International Mathematical Forum, 2, 2007, no. 57, 2841 - 2845

\title{
Intertwining Operators
}

\section{on Banach Function Spaces}

\author{
Bahmann Yousefi \\ Department of Mathematics, College of Sciences \\ Shiraz University, Shiraz 71454, Iran \\ byousefi@shirazu.ac.ir \\ Jinalo Doroodgar \\ Shiraz Farzanegan Pre-University \\ Fars Education Organization, Shiraz, Iran \\ jinalollo-dorodgar@yahoo.com
}

\begin{abstract}
Suppose that $X$ is a Banach space of analytic functions on the open unit disc. Under some conditions we will characterize the operator $T$ : $X \rightarrow X$ intertwining with special multiplication operators.
\end{abstract}

Mathematics Subject Classification: Primary 47B35; Secondary 47B38

Keywords: Banach spaces of analytic functions, bounded point evaluation, multiplication operators

\section{Introduction}

Suppose that the set of analytic polynomials is dense in a Banach space $X$ of functions analytic on the open unit disc $U$, and suppose that for each point $\lambda \in U$ the linear functional of evaluation at $\lambda$ is bounded on $X$. We further assume that $X$ contains the constant functions and multiplication by the independent variable $z$ defines a bounded linear operator $M_{z}$ on $X$. Also, let every $f$ in $X$ has a unique decomposition $f=f_{0} \bigoplus f_{1}$ where $f_{i}$ belongs to $X_{i}$ that is the closed linear span of the set $\left\{z^{2 k+i}: k \geq 0\right\}$ in $X$ for $i=0,1$. 
A complex-valued function $\varphi$ on $\Omega$ for which $\varphi f \in X$ for every $f \in X$ is called a multiplier of $X$, and every multiplier $\varphi$ of $X$ determines a multiplication operator $M_{\varphi}$ on $X$ by $M_{\varphi} f=\varphi f, f \in X$. The set of all multipliers of $X$ is denoted by $M(X)$. Clearly $M(X) \subset H^{\infty}(\Omega)$, where $H^{\infty}(\Omega)$ is the space of all bounded analytic functions on $\Omega$.

\section{Main results}

Let $B(X)$ be the set of all bounded operators on $X$. In the rest of the paper we assume that $X$ is a Banach space of analytic functions on the open unit disc $U$ satisfying the conditions that come in the introduction. For some works on these topics see [1-7]. Assume, further that the composition operators $C_{a \varphi}$ are bounded and invertible on $X$ where $\varphi$ is a multiplier of $X$ and $0<|a| \leq 1$.

Theorem 1. Let $0<|a| \leq 1$ and $T \in B(X)$ be such that $T M_{\varphi^{2}}=a^{2} M_{\varphi^{2}} T$. If $T M_{\varphi}-a M_{\varphi} T$ is compact, then $T C_{\varphi}=M_{u_{0}} C_{a \varphi}$ where $u_{0}=T(1)$.

Proof. Let $u_{0}=T(1)$ and put

$$
u_{1}=\left(T M_{\varphi}-a M_{\varphi} T\right)(1)
$$

For all integers $k \geq 0$ we have

$$
\begin{aligned}
T C_{\varphi} z^{2 k} & =\left(T M_{\varphi}^{2 k}\right)(1) \\
& =\left(a^{2 k} M_{\varphi}^{2 k} T\right)(1) \\
& =u_{0} a^{2 k} C_{\varphi} z^{2 k} \\
& =u_{0} C_{a \varphi} z^{2 k} .
\end{aligned}
$$

Also, by using the relation $(*)$ we get

$$
\begin{aligned}
T C_{\varphi} z=T(\varphi)=\left(T M_{\varphi}\right)(1) & =u_{1}+\left(a M_{\varphi} T\right)(1) \\
& =u_{1}+u_{0} a C_{\varphi} z \\
& =u_{1}+u_{0} C_{a \varphi} z
\end{aligned}
$$

Therefore

$$
\begin{aligned}
T C_{\varphi} z^{2 k+1} & =\left(T M_{\varphi}^{2 k+1}\right)(1) \\
& =\left(T M_{\varphi}^{2 k} M_{\varphi}\right)(1) \\
& =\left(a^{2 k} M_{\varphi}^{2 k} T M_{\varphi}\right)(1) \\
& =a^{2 k} M_{\varphi}^{2 k}\left(u_{1}+u_{0} C_{\varphi} C_{a z} z\right) \\
& =u_{1} C_{\varphi} C_{a z} z^{2 k}+u_{0} C_{\varphi} C_{a z} z^{2 k+1} \\
& =\left(u_{0}+\frac{u_{1}}{a \varphi}\right) C_{a \varphi} z^{2 k+1}
\end{aligned}
$$


for all integers $k \geq 0$. Now consider a polynomial $p$ with decomposition $p=$ $p_{0} \bigoplus p_{1}$, where $p_{i} \in X_{i}$ for $i=0,1$. So we have

$$
\begin{aligned}
T C_{\varphi} p & =T C_{\varphi} p_{0}+T C_{\varphi} p_{1} \\
& =u_{0} C_{a \varphi} p_{0}+\psi_{1} C_{a \varphi} p_{1}
\end{aligned}
$$

where $\psi_{1}=u_{0}+u_{1} / a \varphi$. Therefore

$$
T C_{\varphi} p=u_{0} C_{a \varphi} p+\frac{u_{1}}{a \varphi} C_{a \phi} p_{1} .
$$

But

$$
C_{a \varphi} p_{1}=\frac{C_{a \varphi} p-C_{-a \varphi} p}{2}
$$

thus

$$
T C_{\varphi} p=u_{0} C_{a \varphi} p+\frac{u_{1}}{2 a \varphi}\left(C_{a \varphi} p-C_{-a \varphi} p\right)
$$

for all polynomials $p$. Now since the set of analytic polynomials is dense in $X$, we get

$$
\text { (**) } \quad T C_{\varphi} f=u_{0} C_{a \varphi} f+\frac{u_{1}}{2 a \varphi}\left(C_{a \varphi}-C_{-a \varphi}\right) f
$$

where $u_{0}=T(1)$ and

$$
u_{1}=\left(T M_{\varphi}-a M_{\varphi} T\right)(1) .
$$

Now by using the relation $(* *)$, we have

$$
\begin{aligned}
\left(T M_{\varphi}-a M_{\varphi} T\right) f= & T C_{\varphi}\left(z . f o \varphi^{-1}\right)-a \varphi T C_{\varphi}\left(f o \varphi^{-1}\right) \\
= & u_{0} M_{a \varphi} C_{a \varphi}\left(f o \varphi^{-1}\right)+\frac{u_{1}}{2}\left(C_{a \varphi}+C_{-a \varphi}\right)\left(f o \varphi^{-1}\right) \\
& -\left[u_{0} M_{a \varphi} C_{a \varphi}\left(f o \varphi^{-1}\right)+\frac{u_{1}}{2}\left(C_{a \varphi}-C_{-a \varphi}\right)\left(f o \varphi^{-1}\right)\right] \\
= & u_{1} C_{\psi} f
\end{aligned}
$$

where $\psi=\varphi^{-1} o$ a . Thus

$$
M_{u_{1}} C_{\psi}=T M_{\varphi}-a M_{\varphi} T
$$

and so $M_{u_{1}} C_{\psi}$ is compact. Now by using the Fredholm alternative theorem, we have $u_{1}=0$. Thus by the relation (**), we get $T C_{\varphi}=M_{u_{0}} C_{a \varphi}$. This completes the proof.

Corollary 2. Let $0<|a| \leq 1$ and $T \in B(X)$ be such that $T M_{z^{2}}=a^{2} M_{z^{2}} T$. If $T M_{z}-a M_{z} T$ is compact, then $T=M_{u_{0}} C_{a z}$ where $u_{0}=T(1)$.

Proof. In Theorem 1, put $\varphi(z)=z$.

Corollary 3. Let $T \in B(X)$ be such that $T M_{\varphi^{2}}=M_{\varphi^{2}} T$. If $T M_{\varphi}-M_{\varphi} T$ is 
compact, then $T=M_{u_{0}}$ where $u_{0}=T(1)$.

Proof. In Theorem 1, put $a=1$.

Corollary 4. Let $\varphi$ be odd and $T \in B(X)$ be such that $T M_{\varphi^{2}}=M_{\varphi^{2}} T$. If $T M_{\varphi}+M_{\varphi} T$ is compact, then $T=M_{u_{0}} C_{-z}$ where $u_{0}=T(1)$.

Proof. In Theorem 1, put $a=-1$. Then $T C_{\varphi}=M_{u_{0}} C_{-\varphi}$ where $u_{0}=T(1)$. But $\varphi$ is odd, so $\varphi(-z)=-\varphi(z)$ and we get

$$
\left(C_{-\varphi} f\right)(z)=f(-\varphi(z))=f(\varphi(-z))=\left(C_{-z} C_{\varphi} f\right)(z)
$$

for all $f \in X$. Therefore $T C_{\varphi}=M_{u_{0}} C_{-z} C_{\varphi}$ which implies that $T=M_{u_{0}} C_{-z}$ and so the proof is complete.

Corollary 5. Let $\varphi(i z)=i \varphi(z)$ and let $T \in B(X)$ be such that $T M_{\varphi^{2}}=$ $-M_{\varphi^{2}} T$. If $T M_{\varphi}-i M_{\varphi} T$ is compact, then $T=M_{u_{0}} C_{i z}$ where $u_{0}=T(1)$.

Proof. In Theorem 1, put $a=i$. So we get $T C_{\varphi}=M_{u_{0}} C_{i \varphi}$. But for all $f \in X$ we have

$$
\left(C_{i \varphi} f\right)(z)=f(i \varphi(z))=f(\varphi(i z))=\left(C_{i z} C_{\varphi} f\right)(z) .
$$

Thus $T C_{\varphi}=M_{u_{0}} C_{i z} C_{\varphi}$ which implies that $T=M_{u_{0}} C_{i z}$ where $u_{0}=T(1)$.

\section{References}

[1] P.S. Bourdon and J.H. Shapiro, Spectral synthesis and common cyclic vectors, Michigan Math. J., 37 (1990), 71-90.

[2] S. Richter, Invariant subspaces in Banach spaces of analytic functions, Trans. Amer. Math. Soc., 304 (1987), 585-616.

[3] K. Seddighi and B. Yousefi, On the reflexivity of operators on function spaces, Proc. Amer. Math. Soc., 116 (1992), 45-52.

[4] A. Shields and L. Wallen, The commutants of certain Hilbert space operators, Indiana Univ. Math. J., 20 (1971), 777-788.

[5] B. Yousefi, Multiplication operators on Hilbert spaces of analytic functions, Archive der Mathematik, 83 (6) (2004), 536-539.

[6] B. Yousefi and S. Foroutan, On the multiplication operators on spaces of analytic functions, Studia Mathematica, 168 (2) (2005), 187-191. 
[7] K. Zhu, Irreducible multiplication operators on spaces of analytic functions, J. Operator Theory, 51 (2004), 377-385.

Received: May 6, 2007 